\title{
BMJ Open How are age-related differences in sleep quality associated with health outcomes? An epidemiological investigation in a UK cohort of 2406 adults
}

Andrew Gadie, ${ }^{1}$ Meredith Shafto, ${ }^{2}$ Yue Leng, ${ }^{3}$ Cam-CAN, Rogier A Kievit ${ }^{4}$

To cite: Gadie A, Shafto M, Leng $Y$, et al. How are agerelated differences in sleep quality associated with health outcomes? An epidemiological investigation in a UK cohort of 2406 adults. BMJ Open 2017;7:e014920. doi:10.1136/ bmjopen-2016-014920

- Prepublication history and additional material for this paper are available online. To view these files please visit the journal online (http://dx.doi. org/10.1136/bmjopen-2016014920).

Received 26 October 2016 Revised 7 March 2017 Accepted 8 March 2017

CrossMark

${ }^{1}$ MRC Cognition and Brain Sciences Unit, Cambridge, UK ${ }^{2}$ Department of Psychology, University of Cambridge, Cambridge, UK

${ }^{3}$ Department of Psychiatry, University of California, San Francisco, California, USA ${ }^{4}$ Cambridge Centre for Ageing and Neuroscience (Cam-CAN), University of Cambridge and MRC Cognition and Brain Sciences Unit, Cambridge, UK

Correspondence to Dr Rogier A Kievit; rogier.kievit@mrc-cbu.cam. ac.uk

\section{ABSTRACT}

Objectives To examine age-related differences in selfreported sleep quality and their associations with health outcomes across four domains: physical health, cognitive health, mental health and neural health.

Setting Cambridge Centre for Ageing and Neuroscience (Cam-CAN) is a cohort study in East Anglia/England, which collected self-reported health and lifestyle questions as well as a range of objective measures from healthy adults.

Participants 2406 healthy adults (age 18-98) answered questions about their sleep quality (Pittsburgh Sleep Quality Index (PSQI)) and measures of physical, cognitive, mental and neural health. A subset of 641 individuals provided measures of brain structure.

Main outcome measures PSQI scores of sleep and scores across tests within the four domains of health. Latent class analysis (LCA) is used to identify sleep types across the lifespan. Bayesian regressions quantify the presence, and absence, of relationships between sleep quality and health measures.

Results Better self-reported sleep is generally associated with better health outcomes, strongly so for mental health, moderately for cognitive and physical health, but not for sleep quality and neural health. LCA identified four sleep types: 'good sleepers' (68.1\%, most frequent in middle age), 'inefficient sleepers' (14.01\%, most frequent in old age), 'delayed sleepers' $(9.28 \%$, most frequent in young adults) and 'poor sleepers' (8.5\%, most frequent in old age). There is little evidence for interactions between sleep quality and age on health outcomes. Finally, we observe $\mathrm{U}$-shaped associations between sleep duration and mental health (depression and anxiety) as well as self-reported general health, such that both short and long sleep were associated with poorer outcomes.

Conclusions Lifespan changes in sleep quality are multifaceted and not captured well by summary measures, but instead should be viewed as as partially independent symptoms that vary in prevalence across the lifespan. Better self-reported sleep is associated with better health outcomes, and the strength of these associations differs across health domains. Notably, we do not observe associations between self-reported sleep quality and white matter.
Strengths and limitations of this study

- Broad phenotypic assessment of healthy ageing across multiple health domains.

- Advanced analytic techniques (ie, latent class analysis regression) allows new insights.

- A uniquely large neuroimaging sample combined with Bayesian inference allows for quantification of evidence for the null hypothesis.

- Subjective sleep measures may have drawbacks in older samples.

- Cross-sectional data precludes modelling of within subject changes.

\section{BACKGROUND}

Sleep is a fundamental human behaviour, with humans spending almost a third of their lives asleep. Regular and sufficient sleep has been shown to benefit human physiology through a number of different routes, ranging from consolidation of memories ${ }^{1}$ to removal of free radicals ${ }^{2}$ and neurotoxic waste. ${ }^{3}$ Sleep patterns are known to change across the lifespan in various ways, including decreases in quantity and quality of sleep, ${ }^{4}$ with up to $50 \%$ of older adults report difficulties initiating and/or maintaining sleep. ${ }^{5}$ A meta-analysis of over 65 studies reflecting 3577 subjects across the lifespan reported a complex pattern of changes, including an increase of stage 1 but a decrease of stage 2 sleep in old age, as well as a decrease in rapid eye movement (REM) sleep. ${ }^{6}$ An epidemiological investigation of self-reported sleep in older adults observed marked sex differences in age-related sleep changes, with females more likely to report disturbed sleep onset but men more likely to report night-time awakenings. ${ }^{7}$ Other findings are age-related physiological changes in the alignment of homeostatic and circadian rhythms, ${ }^{8}$ 
decreases in sleep efficiency, ${ }^{9}$ the amount of slow-wave sleep and an increase in daytime napping. ${ }^{10}$ Importantly, interruption and loss of sleep have been shown to have wide-ranging adverse effects on health ${ }^{11}$ leaving open the possibility that age-related changes in sleep patterns and quality may contribute to well-documented age-related declines in various health domains.

In the current study, we examine self-reported sleep habits in a large, population-based cohort Cambridge Centre for Ageing and Neuroscience $\left(\mathrm{Cam}-\mathrm{CAN}^{12}\right)$. We relate sleep measures to measures of health across four health domains: cognitive, brain health, physical and mental health. Our goal is to quantify and compare the associations between typical age-related changes in sleep quality and a range of measures of health measures that commonly decline in later life. We assess sleep using a self-reported measure of sleep quality, the Pittsburgh Sleep Quality Index (PSQI). ${ }^{13}$ The PSQI has good psychometric properties ${ }^{14}$ and has been shown to correlate reliably with diseases of ageing and mortality. ${ }^{15-17}$ Although polysomnography ${ }^{18}$ is commonly considered the gold standard of sleep quality measurement, it is often prohibitively challenging to employ in large samples. A recent direct comparison of sleep measures ${ }^{19}$ suggests that although subjective sleep measures (such as PSQI) may have certain drawbacks in older samples, they also capture complementary aspects of sleep quality not fully captured by polysomnography. Moreover, collecting self-report sleep quality data in a large, deeply phenotyped cohort offers several additional benefits.

By using a population cohort of healthy adults and studying a range of health outcomes in the same population, we can circumvent challenges associated with studying clinical populations and provide new insights. First and foremost, by investigating associations between sleep and outcomes across multiple health domains in the same sample, we can make direct comparisons of the relative magnitude of these effects. Second, larger samples allow us to generate precise effect size estimates, as well as adduce evidence in favour of the null hypothesis. Third, we investigate the associations between sleep quality and neural health in a uniquely large healthy population. Previous investigations of the consequences of poor sleep, especially on neural health, generally focused on clinical populations such as those suffering from insomnia. ${ }^{20}{ }^{21}$ Although such studies are crucial for understanding pathology, the demographic idiosyncrasies and often modest sample sizes of these approaches make it hard to generalise to healthy, community-dwelling lifespan populations. Moreover, most studies that study age-related changes or differences focus on (very) old age, while far less is known about young and middle-aged adults. ${ }^{6}$ For these reasons, our focus on a healthy, multimodal lifespan cohort is likely to yield novel insights into the subtle changes in sleep quality across the lifespan.

We will focus on three questions within each health domain. First, is there a relationship between sleep quality and health? Second, does the strength and nature of this relationship change when age is included as a covariate? Third, does the strength and nature of the relationship change across the lifespan? We will examine these questions across each of the four health domains.

\section{METHODS \\ Sample}

A cohort of $2544^{12}$ was recruited as part of the population-based Cam-CAN cohort (www.cam-can.com), drawn from the general population via Primary Care Trust's (PCT) lists within the Cambridge City (UK) area; 10520 invitation letters were sent between 2010 and 2012, and willing participants were invited to have an interview conducted in their home, with questions on health, lifestyle demographics and core cognitive assessments. Sample size was chosen to allow for 100 participants per decile in further acquisition stages, giving sufficient power to separate age-related change from other sources of individual variation. For additional details of the project protocol, see Shafto et $a l^{12}$ and Taylor $e t a l^{22}$ and for further details of the Cam-CAN dataset visit http://www.mrc-cbu.cam.ac.uk/datasets/ camcan/. A further subset of participants who were MRI compatible with no serious cognitive impairment participated in a neuroimaging session ${ }^{22}$ between 2011 and 2013. Participants included were native English speakers, had normal or corrected to normal vision and hearing, and scored 25 or higher on the Mini-Mental State Examination (MMSE). ${ }^{23}$ Note that other, more stringent cut-offs are sometimes employed to screen for premorbid dementia, such as a score of 88 or higher in the Addenbrooke's Cognitive Examination Revised (ACE-R). ${ }^{24}$ For the sake of comprehensiveness, we repeated our analyses using this more stringent cut-off (ACE-R >88), but observed no noteworthy differences in our findings, so we only report the findings based on the MMSE exclusion criteria. Ethical approval for the study was obtained from the Cambridgeshire 2 (now East of England-Cambridge Central) Research Ethics Committee (reference: 10/H0308/50). Participants gave written informed consent. The raw data and analysis code are available on signing a data sharing request form (see http://www.mrc-cbu.cam.ac.uk/datasets/ camcan/ for more detail).

\section{Variables}

\section{Sleep measures}

Sleep quality was assessed using the PSQI, a well-validated self-report questionnaire ${ }^{13} 19$ designed to assist in the diagnosis of sleep disorders. The questions concern sleep patterns, habits and lifestyle questions, grouped into seven components, each yielding a score ranging from 0 (good sleep/no problems) to 3 (poor sleep/severe problems), that are commonly summed to a PSQI total score ranging between 0 and 21, with higher scores reflecting poorer sleep quality. 
Health measures

\section{Cognitive health}

A number of studies have found associations between poor sleep and cognitive decline, including in elderly populations. Poor sleep affects cognitive abilities such as executive functions ${ }^{25}$ and learning and memory processes, ${ }^{26}$ whereas short-term pharmaceutical interventions such as administration of melatonin improve both sleep quality and cognitive performance. ${ }^{2728}$ Recent work $^{29}$ concluded that 'maintaining good sleep quality, at least in young, adulthood and middle age, promotes better cognitive functioning and serves to protect against age-related cognitive declines'. As sleep may affect various aspects of cognition differently, ${ }^{30}$ we include measures that cover a range of cognitive domains including memory, reasoning, response speed and verbal fluency, as well a measure of general cognition (see table 1 and Shafto et $a l^{12}$ for more details).

\section{Neural health}

Previous research suggests that individuals with a severe disruption of sleep are significantly more likely to exhibit signs of poor neural health. ${ }^{20}{ }^{31}$ Specifically, previous studies have observed decreased white matter health in clinical populations suffering from conditions such as chronic insomnia, ${ }^{21}$ obstructive sleep apnoea, ${ }^{32} 33$ excessively long sleep in patients with diabetes ${ }^{34}$ and REM sleep behaviour disorder. ${ }^{35}$ Many of these studies focus on white matter hyperintensities (WMHs), a measure of the total volume or number of regions showing low-level neural pathology (although some study grey matter, eg, Macey et $a l^{36}$ and Sexton et $a l^{37}$ ). WMHs are often used as a clinical marker, as longitudinal increases in WMHs are associated with increased risk of stroke, dementia and death $^{38}$ and are more prevalent in patients with pathological sleep problems. ${ }^{33} 34$ However, use of this metric in clinical cohorts largely leaves open the question of the impact of sleep quality on neural (white matter) health in non-clinical, healthy populations. To address this question, we use a more general indicator of white matter neural health; fractional anisotropy (FA). FA is associated with white matter integrity and myelination. ${ }^{39}$ We use FA as recent evidence suggests that WMHs represent the extremes (foci) of white matter damage and that FA is able to capture the full continuum of white matter integrity. ${ }^{41}$ For more information regarding the precise white matter pipeline, see Shafto et $a l^{12}$, Taylor $e t a l^{22}$ and Kievit et $a l^{42}$

\section{Physical health}

Sleep quality is also an important marker for physical health, with poorer sleep being associated with conditions such as obesity, diabetes mellitus, ${ }^{43}$ overall health $^{1144}$ and increased all-cause mortality. ${ }^{45} 46$ We focus on a set of variables that capture three types of health domains commonly associated with poor sleep: cardiovascular health measured by pulse, systolic and diastolic blood pressure, ${ }^{47}$ self-reported health, both in general and for the past 12 months $^{48}$ and body mass index. $^{49}$

\section{Mental health}

Previous work has found that disruptions of sleep quality are a central symptom of forms of psychopathology such as major depressive disorder, including both hypersomnia and insomnia, ${ }^{450}$ and earlier episodes of insomnia greatly increased the risk of later episodes of major depression. ${ }^{51}$ Kaneita $e t a \tilde{l}^{2}$ found a U-shaped association between sleep and depression, such that individuals regularly sleeping less than 6 hours, or more than 8 hours, were more likely to be depressed. Both depression ${ }^{53}$ and anxiety ${ }^{5455}$ are commonly associated with sleep problems. To capture these dimensions, we used both scales of the Hospital Anxiety and Depression Scale (HADS), ${ }^{56}$ a widely used and standardised questionnaire that captures self-reported frequency and intensity of anxiety and depression symptoms.

\section{STATISTICAL ANALYSES}

We examined whether self-reported sleep patterns change across the lifespan, both for the PSQI sum score and for each of the seven PSQI components. We then examined the relationships between the sleep quality and the four health domains in three ways. First, simple regression of the health outcome on sleep variables to determine evidence for association between poor sleep quality and poor health outcomes. Second, we included age as a covariate. Finally, we included a (standard normal rescaled) continuous interaction term to examine whether there is evidence for a changing relationship between sleep and outcomes across the lifespan.

For all regressions, we used a default Bayesian approach advocated by Liang et $a l^{57}$, Rouder and Morey, ${ }^{58}$ Wagenmakers $^{59}$ and Wetzels et a ${ }^{60}$ which avoids several well-documented issues with p-values, ${ }^{59}$ allows for quantification of null effects and decreases the risk of multiple comparison problems. ${ }^{61}$ Bayesian regressions allows us to symmetrically quantify evidence in favour of, or against, some substantive model as compared with a baseline (eg, null) model. This evidential strength is expressed as a Bayes factor, ${ }^{62}$ which can be interpreted as the relative likelihood of one model versus another given the data and a certain prior expectation. A Bayes factor of, for example, 7 in favour of a simple linear regression model suggests that the data are seven times more likely under that model than an intercept only model for a given prior (for an empirical comparison of p-values and Bayes factors, see Wetzels et $\left.a l^{60}\right)$. A heuristic summary of evidential interpretation can be seen in figure 1 .

We report log Bayes factors for (very) large effects and regular Bayes factors for smaller effects. To compute Bayes factors, we used default Bayes factor approach for model selection ${ }^{57} 58$ in the package BayesFactor ${ }^{63}$ using the open source software package $\mathrm{R} .{ }^{64}$ As previous papers report associations between sleep and outcomes ranging 


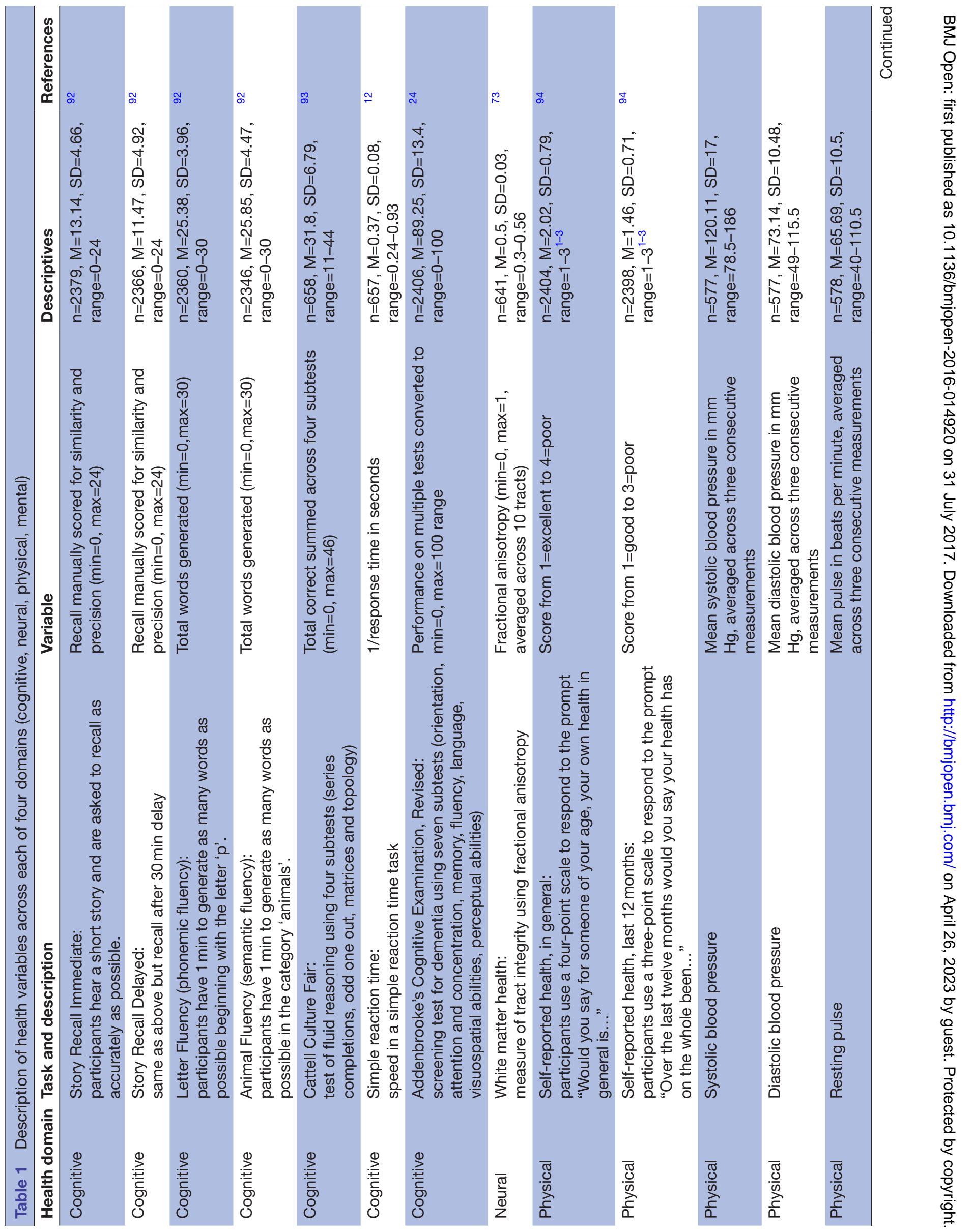




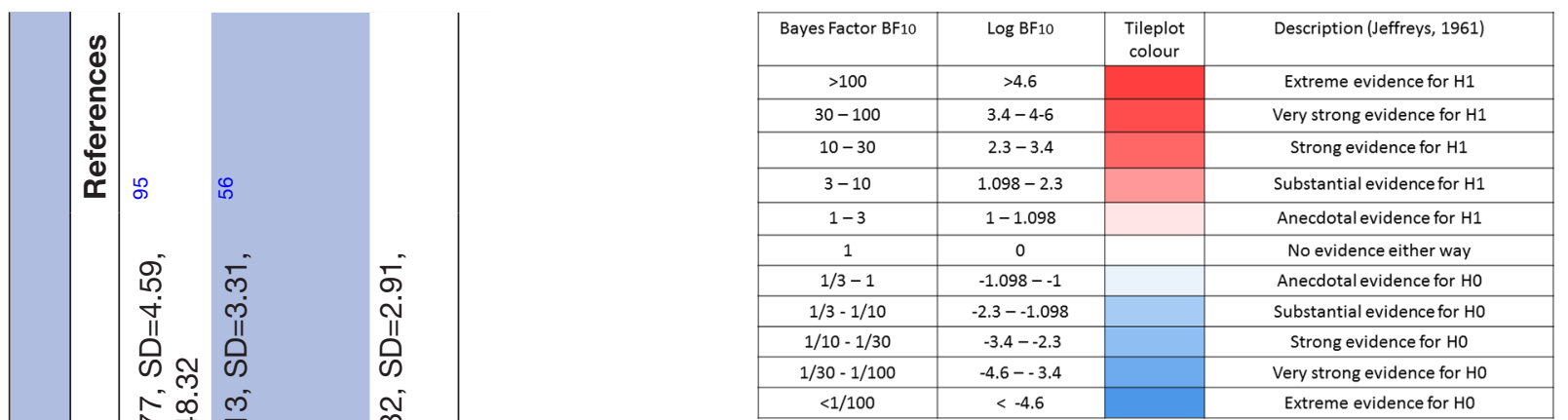

Figure 1 Descriptive interpretation of Bayes factors.

from absent to considerable in size, we used the default, symmetric Cauchy prior with width $\frac{\sqrt{2}}{2}$ which translates to a $50 \%$ confidence that the true effect will lie between -0.707 and 0.707 . Prior to further analysis, scores on all outcomes were transformed to a standard normal distribution, and any scores exceeding a z-score of 4 or -4 were recoded as missing (aggregate percentage outliers across the four health domains: cognitive, $0.41 \%$; mental, $0.16 \%$; neural, $0.37 \%$; physical, $0.031 \%$ ).

\section{RESULTS}

\section{Age-related differences in sleep quality}

First, we examined sleep changes across the lifespan by examining age-related differences in the PSQI sum score $(\mathrm{n}=2178, \mathrm{M}=5.16, \mathrm{SD}=3.35$, range $=0-19)$. Regressing the PSQI global score on age (see online supplementary figure 1) showed evidence for a positive relationship across the lifespan $\left(\log B \mathrm{~F}_{10}=10.45\right)$. This suggests that on the whole, sleep quality decreases across the lifespan (note that higher PSQI scores correspond to worse sleep). Although we observed strong statistical evidence for an age-related difference ('extreme' according to Jeffreys ${ }^{62}$ ), age explained only $1.23 \%$ of the variance in the PSQI total score. Next, we regressed each of the seven components on age in the same manner. In online supplementary figure 2, we see that age has varying and specific effects on different aspects of sleep quality and did not worsen uniformly across the lifespan. For example, we observed moderate evidence that sleep latency did not change across the lifespan (sleep latency, $\mathrm{BF}_{01}=9.25$, in favour of the null), sleep quality showed no evidence for either change or stasis $\left(\mathrm{BF}_{10}=1.63\right)$ and one sleep component, daytime dysfunction, improved slightly across the lifespan $\left(\mathrm{BF}_{10}=7.03\right)$. The strongest age-related decline is that of efficiency, showing an r-squared of $6.6 \%$.

Finally, we entered all seven components into a Bayesian multiple regression simultaneously to examine to what extent they could, together, predict age. The best model included every component except sleep latency $\left(\log \mathrm{BF}_{10}=142.71\right)$. Interestingly, this model explained $13.66 \%$ of the variance in age, compared with $1.23 \%$ for the PSQI total score and $6.6 \%$ for the strongest single component (efficiency). This shows that lifespan changes in self-reported sleep are heterogeneous and partially 
independent and that specific patterns and components need to be taken into account simultaneously to fully understand age-related differences in sleep quality. These findings show that neither the PSQI sum score nor the sleep components in isolation fully capture differences in sleep quality across the lifespan.

The analysis above suggests that conceptualising 'poor sleep' as a single dimension does not reflect the subtleties in lifespan changes-an often computed sumscore changes little across the lifespan, whereas the totality of sleep symptoms shows far stronger, and more subtle, patterns. To better elucidate individual differences in sleep quality, we next use latent class analysis (LCA). ${ }^{65}$ This technique will allow us examine individual differences in sleep quality across the lifespan in more detail than afforded by simple linear regressions: rather than examining continuous variation in sleep components, LCA classifies individuals into different sleep types, each associated with a distinct profile of 'sleep symptoms'. If there are specific constellations of sleep problems across individuals, we can quantify and visualise such sleep types.

To analyse the data in this manner, we binarised the responses on each component into 'good' ( 0 or 1$)$ or 'poor' (2 or 3). Our measures of PSQI symptoms straddle the border between continuous and categoricalalthough some are fully continuous (eg, sleep latency) others are less so. For instance, although scored on a range of four several of the scales (such as Subjective Sleep quality) have implicitly binary response options of 'very good' and 'fairly good' on the one hand and 'fairly bad' and 'very bad' on the other. As analytical work in psychometrics $^{66}$ suggests that Likert-like graded scales can be treated as continuous only from five ordinal categories upwards, by fitting an LCA we are erring on the side of caution (although a latent profile analysis would likely give similar results). Note that although our analysis divides individuals into discrete classes with specific profiles, it is still possible to examine the conditional response likelihood of responding 'yes' to each symptom as a continuous metric (between 0 and 1) that reflects the nature of the association between the class and the outcome. By modelling sleep 'types', we hope to illustrate the complex patterns in a more intelligible mannernotably, doing so allows us to examine whether the likelihood of belonging to any sleep 'type' changes as a function of age.

Next, we examined evidence for distinct sleep types using a set of possible models (varying from 2 to 6 sleep types). We found that the four-class solution gives the best solution, according to the Bayesian Information Criterion $^{67}$ (BIC for four classes $=11825.65$, lowest BIC for other solutions $=11884.92$ (five classes) (with 50 repetitions per class, at 5000 maximum iterations)). Next, we inspected the nature of the sleep types, the prevalence of each 'sleep type' in the population and whether the likelihood of belonging to a certain sleep type changes across the lifespan. See figure 2 for the component profiles of the four sleep types identified.
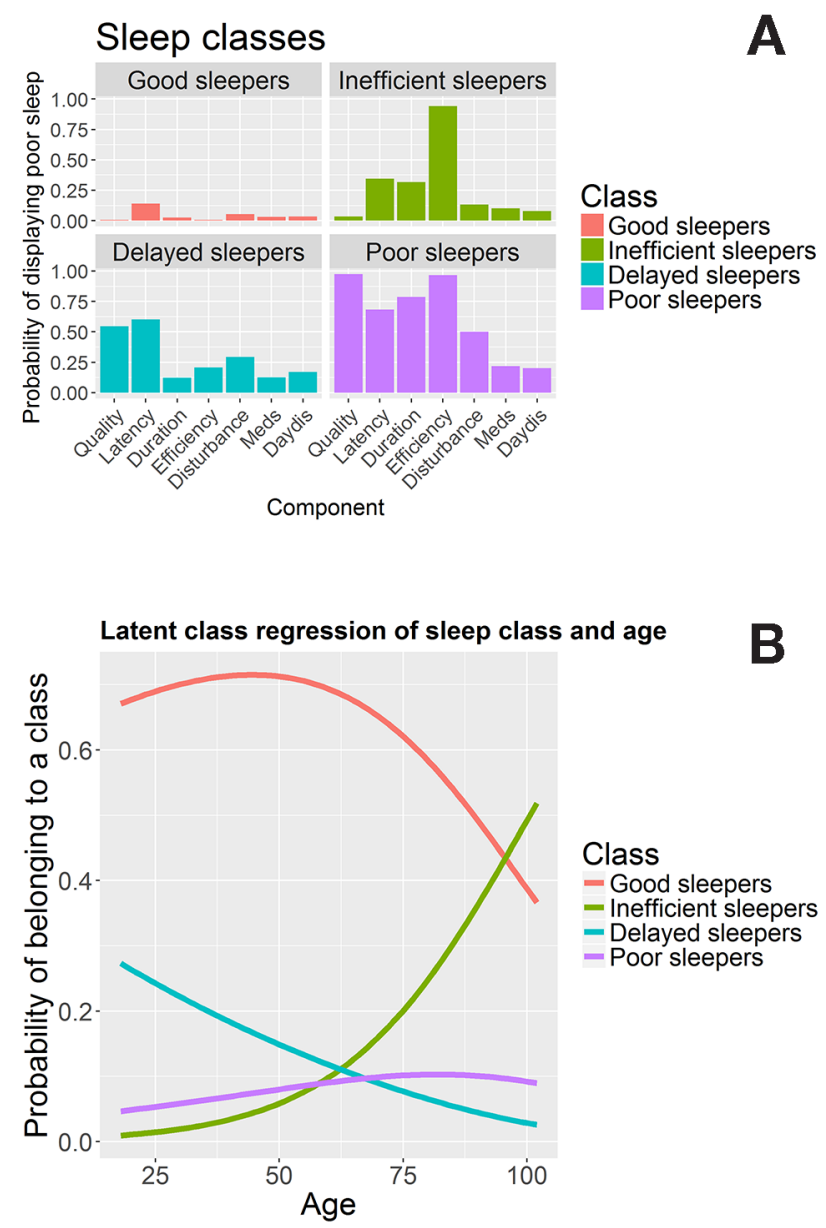

Figure 2 Latent class analysis. Panel (A) shows the sleep quality profiles for each of the four classes. Panel (B) shows the conditional probability of belonging to each class across the lifespan.

Class 1, 'good sleepers', makes up $68.1 \%$ of participants. Their sleep profile is shown in figure 2A, top left, and is characterised by a low probability of responding 'poor' to any of the sleep components. Class 2, 'inefficient sleepers', makes up $14.01 \%$ of the participants and is characterised by poor sleep efficiency: members of this group uniformly $(100 \%)$ report poor sleep efficiency, despite relatively low prevalence of other sleep problems, as seen in figure 2A, top right. Class 3, 'delayed sleepers' seen in the bottom left of figure $2 \mathrm{~A}$, makes up $9.28 \%$ of the participants: characterised by modestly poor sleep across the board, but a relatively high probability of poor scores on sleep latency (59\%), sleep quality $(51 \%)$ and sleep disturbance $(31 \%)$. Finally, class 4 , 'poor sleepers', makes up $8.5 \%$ of the participants, shown bottom right in figure 2A. Their responses to any of the seven sleep components are likely to be 'poor' or 'very poor', almost universally so for 'sleep quality' (94\%) and 'sleep efficiency' $(97.7 \%)$.

Next, we included age as a covariate (simultaneously including a covariate is known as latent class regression or concomitant-variable latent class models). ${ }^{68}$ This analysis, 
visualised in figure $2 \mathrm{~B}$, shows that the probability of membership of each classes compared with the reference class (good sleepers) changes significantly across the lifespan for each of the classes (class 2 vs class 1: beta/ $\mathrm{SE}=0.05 / 0.00681, \mathrm{t}=7.611$; class 3 vs class 1 : beta/ $\mathrm{SE}=-0.01948 / 0.0055, \mathrm{t}=-3.54$; class 4 vs class 1 : beta $/ \mathrm{SE}$ $0.01269 / 0.00478, \mathrm{t}=2.655$ ), for more details on generalised logit coefficients, see Linzer and Lewis. ${ }^{65}$ The frequency of class 1 (good sleepers) peaks in middle to late adulthood, dropping increasingly quickly after age 50 . Class 2 (inefficient sleepers) are relatively rare in younger individuals, but the prevalence increases rapidly in individuals over age 50. On the other hand, class 3 (delayed sleepers) shows a steady decrease in the probability of an individual showing this profile across the lifespan, suggesting that this specific pattern of poor sleep is more commonly associated with younger adults. Finally, the proportion of class 4 (poor sleepers) members increases only slightly across the lifespan. Together, the LCA provides additional evidence that the PSQI sum score as an indicator of sleep quality does not fully capture the subtleties of age-related differences. Age-related changes in sleep patterns are characterised by specific, clustered patterns of sleep problems that cannot be adequately characterised by summation of the component scores. The above analyses show how both a summary measure and individual measures of sleep quality change across the lifespan. Next, we examined the relationships between sleep quality measures (seven components and the global PSQI score) and health variables (specific variables across four domains, as shown in table 1 ).

\section{Sleep, health domains and age}

Cognitive health

First, we examined the relationships between sleep quality and seven measures of cognitive health (see table 1 for details). We visualise our findings using tileplots. ${ }^{69}$ Each cell shows the numeric effect size (r-squared, 0-100) of the bivariate association between a sleep component and a health outcome, colour coded by the statistical evidence for a relationship using the Bayes factor. If the parameter estimate is positive, the r-squared value has the symbol ' + ' added (note the interpretation depends on the nature of the variable, cf. table 1 ).

As can be seen in online supplementary figure 3, several relationships exist between measures of cognitive health and measures of sleep quality. However, these results attenuate in a multiple regression model including age as shown in figure 3 .

The cognitive abilities most strongly associated with poor sleep are a measure of general cognitive health, ACE-R and a test of verbal phonemic fluency. Two patterns emerged. First, the strongest predictor across the simple and multiple regressions was for the PSQI total score. Tentatively, this suggests that a cumulative index of sleep problems, rather than any specific pattern of poor sleep, is the biggest risk factor for poorer cognitive performance. Second, after controlling for age, the most

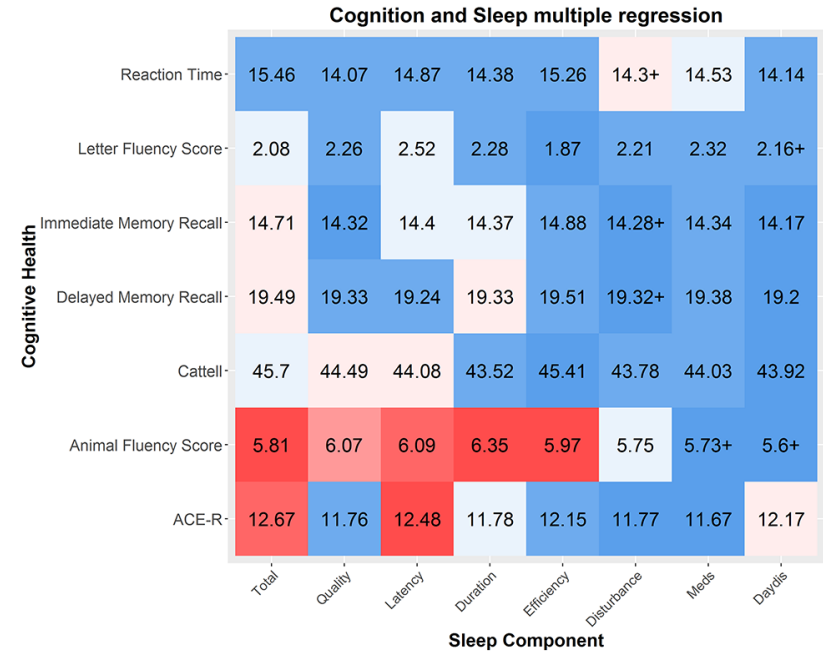

Figure 3 Multiple regressions between sleep components and cognitive health. The strength of the effect is colour coded by Bayes factor and the effect size is shown as $r$-squared (as a percentage out of 100). Sample varies across components and measures due to varying missingness. Cattell and reaction time were measured only in the imaging cohort: mean $\mathrm{N}=648, \mathrm{~N}=11.11$. Sample sizes for five other domains are similar (mean $n=2300.25, S D=65.57$ ). ACE-R, Addenbrooke's Cognitive Examination Revised.

strongly affected cognitive measure is phonemic fluency, the ability to generate name as many different words as possible starting with a given letter within a minute. Verbal fluency is commonly used as a neuropsychological test. ${ }^{70}$ Previous work suggests that it depends on both the ability to cluster (generating words within a semantic cluster) and to switch (switching between categories) and is especially vulnerable to frontal and temporal lobe damage (with specific regions dependant on either a semantic or phonemic task ${ }^{71}$ ). Although modest in size, our findings suggests this task, dependent on multiple executive processes, is particularly affected by poor sleep quality. ${ }^{72}$ The second strongest association was with the ACE-R, a general cognitive test battery similar in style and content to the MMSE. Little evidence for interactions with age was observed (mean $\log \mathrm{BF}_{10}=-2.08$, see online supplementary figure 4), suggesting that the negative associations between sleep and cognitive performance are a constant feature across the lifespan, rather than specifically in elderly individuals. Together, this suggests that poor sleep quality is modestly but consistently associated with poorer general cognitive performance across the lifespan, most strongly with semantic fluency.

\section{Neural health}

Using diffusion tensor imaging, we estimated a general index of white matter integrity in 10 tracts $^{73}$ (shown in online Supplementary figure 5), by taking the average FA in each white matter region of interest (ROI) (see Kievit et $a t^{42}$ for more information). We used the data from a subsample of 641 individuals (age $\mathrm{M}=54.87$, range 18.4888.96 ) who were scanned in a 3 T MRI scanner (for more 


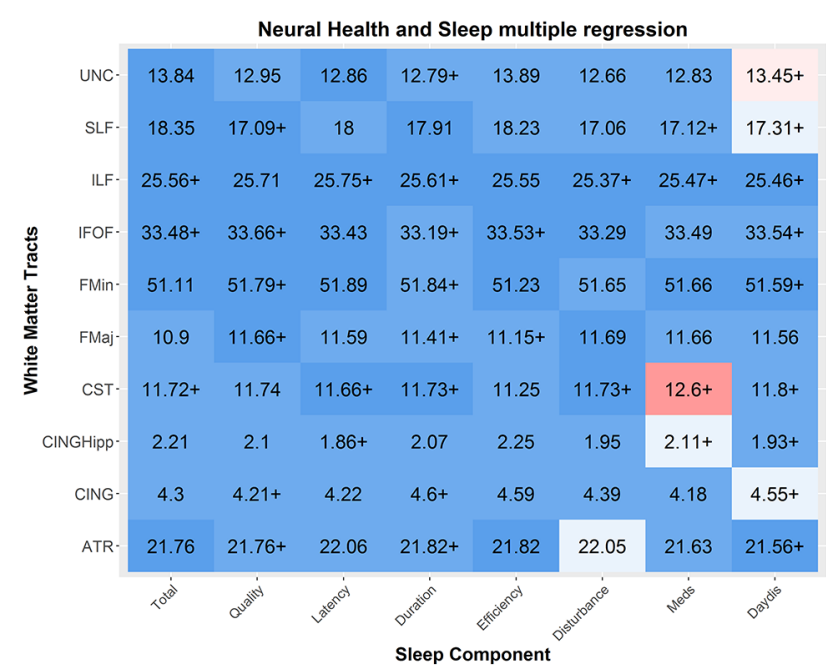

Figure 4 Multiple regressions between sleep components and neural health. Each cell represents the relationship between a sleep component and the mean neural health in a given tract as index by fractional anisotrophy. Numbers represented in r-squared. White matter tracts abbreviations: uncinate fasciculus (UNC), superior longitudinal fasciculus (SLF), inferior longitudinal fasciculus (ILF), inferior frontooccipital fasciculus (IFOF), forceps minor (FMin), forceps major (FMaj), cerebrospinal tract (CST), the ventral cingulate gyrus (CINGHipp), the dorsal cingulate gyrus (CING) and the anterior thalamic radiations (ATR). $\mathrm{N}$ varies slightly across components due to varying missingness ( $\mathrm{N}$ mean=631.325, $\mathrm{SD}=10.32$ ).

details regarding the pipeline, sequence and processing steps, see Taylor et $a l^{22}$ and Kievit et $\left.a t^{42}\right)$. Regressing neural white matter ROI's on sleep quality, we find several small effects, with the strongest associations between sleep efficiency and neural health (see online supplementary figure 6). All effects are such that poorer sleep is associated with poorer neural health, apart from a small effect in the opposite direction for uncinate and daytime dysfunction $\left(\mathrm{BF}_{10}=6.20\right)$. However, when age is included as a covariate, the negative associations between sleep quality and white matter health are attenuated virtually to zero (figure 4, mean/median $\mathrm{BF}_{10}=0.18 / .10$ ), with Bayes factors providing strong evidence for the lack of associations between sleep quality and white matter integrity. One exception was observed: the use of sleep medication is associated with better neural health in the corticospinal tract, a region previously found to be affected by pathological sleep problems such as sleep apnoea. ${ }^{33}$ However, this effect is very small $\left(\mathrm{BF}_{10}=3.24\right)$ given the magnitude of the sample and the range of comparisons, so should be interpreted with caution.

Finally, we tested for any interactions by including a mean-scaled interaction term (sleep*age, see online supplementary figure 7). This analysis found evidence for a significant interaction, between the superior longitudinal fasciculus (SLF) and sleep medication $\left(\mathrm{BF}_{10}=13.77\right)$, such that better neural health in the SLF was associated with the use of sleep medication more strongly in older adults. Together, these findings suggest that in general, once age

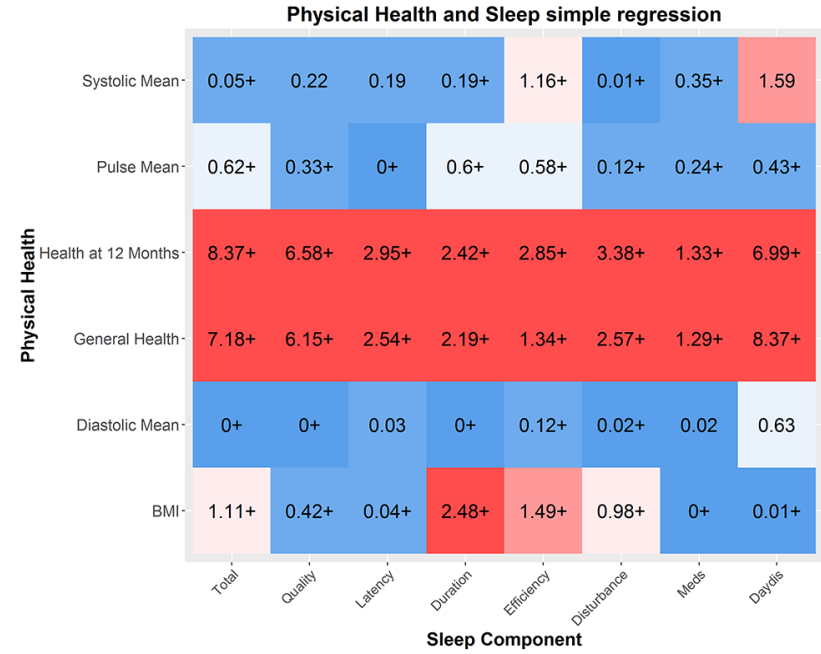

Figure 5 Physical health and sleep quality. Numbers represent r-squared. Strong associations between general indices of health and sleep quality are found, and several modest relationships with BMI and sleep quality. Selfreported health (12 months and general) were measured in the full cohort (mean=2315.37, SD=66.29), the other indicators were measured in the imaging cohort only (mean=569.87, SD=11.16). BMI, body mass index.

is taken into account, self-reported sleep problems in a non-clinical sample are not associated with poorer neural health, although there is some evidence for a modest associations between better neural health in specific tracts and the use of sleep medication in the elderly.

\section{Physical health}

Next, we examined whether sleep quality is associated with physical health. Figure 5 shows the simple regressions between sleep quality and physical health. Strong associations were found between poor overall sleep (PSQI sum score) and poor self-reported health, both in general $\left(\operatorname{logBF}{ }_{10}=77.51\right)$ and even more strongly for health in the past 12 months $\left(\log \mathrm{BF}_{10}=91.25\right)$. This may be because poorer sleep, across all components, directly affects general physical health ${ }^{43}$ or because people subjectively experience sleep quality as a fundamental part of overall general health. A second association was between BMI and poor sleep quality, most strongly poor duration $\left(\log \mathrm{BF}_{10}=4.69\right)$.

This not only replicates previous findings but is in line with an increasing body of evidence that suggests that short sleep duration causes metabolic changes, which in turn increases the risk of both diabetes mellitus and obesity. ${ }^{437576}$ Next, we examined whether these effects were attenuated once age was included. We show that although the relationships are slightly weaker, the overall pattern remains (see online supplementary figure 8), suggesting that these associations are not merely co-occurrences across the lifespan. Our findings suggest self-reported sleep quality, especially sleep duration, is 
related to differences in physical health outcomes in a healthy sample.

Finally, there was evidence of a single interaction with age (see online supplementary figure 9). Although poor sleep duration was associated with higher diastolic blood pressure in younger adults, it was associated with lower diastolic blood pressure in older individuals $\left(\mathrm{BF}_{10}=8.53\right)$. This may reflect the fact that diastolic blood pressure is related to cardiovascular health in a different way across the lifespan, although given the small effect size it should be interpreted with caution.

\section{Mental health}

Finally, we examined the relationship between sleep quality and mental health, as measured by the HADS. ${ }^{56}$ One benefit of the HADS in this context is that, unlike some other definitions (eg, the Diagnostic and Statistical Manual of Mental Disorders-V), sleep quality is not an integral (scored) symptom of these dimensions. As shown in online supplementary figure 10, there are very strong relationships between all aspects of sleep quality and measures of both anxiety and depression. The strongest predictors of depression are daytime dysfunction $\left(\log \mathrm{BF}_{10}=245.9, \mathrm{r}^{2}=19.26 \%\right)$, followed by the overall sleep score $\left(\log \mathrm{BF}_{10}=170.5, \mathrm{r}^{2}=14.92 \%\right)$ and sleep quality $\left(\log \mathrm{BF}_{10}=106.8, \mathrm{r}^{2}=8.9 \%\right)$. The effects size for anxiety was comparable but slightly smaller in magnitude. When age is included as a covariate, the relationships remained virtually unchanged (see online supplementary figure 11), suggesting that these relationships are present throughout across the lifespan. These findings replicate and extend previous work, suggesting that sleep quality is strongly associated with both anxiety and depression across the lifespan.

Finally, we examined a model with an interaction term (see online supplementary figure 12). Most prominently, we found interactions with age in the relationship between HADS anxiety and the PSQI total, such that for the relationship between anxiety and overall sleep quality

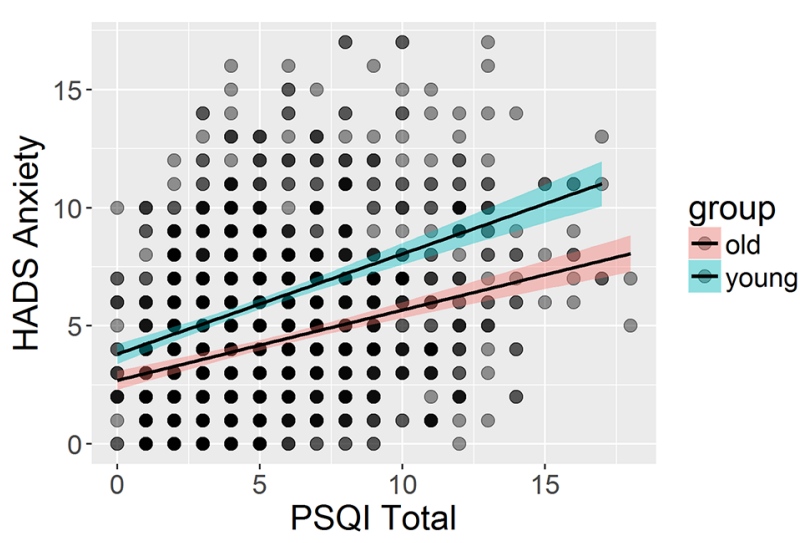

Figure 6 Interaction between sleep quality and anxiety in the youngest third $(n=723$, age 18.48-46.2) compared with the oldest third of participants $(n=724$, age 71.79-98.88). HADS, Hospital Anxiety and Depression Scale; PSQI, Pittsburgh Sleep Quality Index. is stronger in younger adults $\left(\mathrm{BF}_{10}=4.6\right.$, see figure 6$)$. Together, our findings show that poor sleep quality is consistently, strongly and stably associated with poorer mental health across the adult lifespan.

Non-linear associations between sleep and health outcomes In the above analyses, we focused on linear associations between symptoms and health outcomes. However, for one aspect of sleep, namely, sleep duration (in hours), evidence exists that these associations are likely to be non-linear, such that both shorter and longer than average sleep are associated with poorer health outcomes (eg, Grandner and Drummond, ${ }^{77}$ Kaneita et $a l^{78}$ and Grandner $e t a l^{79}$ ). This is echoed in clinical criteria for depression, which commonly include both hypersomnia and hyposomnia as 'sleep disruption' symptoms. In other words, both too much and too little sleep are suboptimal. To examine whether we observe evidence for non-linearities, we examined the relationship between raw scores on sleep duration (in hours, not transformed to PSQI norms) and health outcomes across the four domains. If the association between sleep and outcomes is indeed U-shaped (or inverted U, depending on the scale), then a Bayesian regression would prefer the less parsimonious model that includes the quadratic term. We observed no non-linear associations between any neural or cognitive health variables. We found strong evidence for a quadratic (subscript q) over a linear (subscript 1) associations between sleep duration and HADS anxiety $\left(\log \mathrm{BF}_{\mathrm{ql}}=19.98\right)$, even more strongly so with HADS depression $\left(\operatorname{logBF} \mathrm{q}_{\mathrm{q}}=26.41\right.$, figure $7 \mathrm{~A}$ shows the strongest curvilinear association, namely with depression). We found a similar U-shaped curve with general health $\left(\mathrm{BF}_{\mathrm{ql}}=277.81\right)$ and self-reported health over the last 12 months $\left(\mathrm{BF}_{\mathrm{q}}=887.6\right)$, the former shown in figure 7B. Together, these analyses support previous conclusions that some (although not all) poorer health outcomes can be associated with both too much and too little sleep.

\section{DISCUSSION}

In this study, we report on the associations between age-related differences in sleep quality and health outcomes in a large, age-heterogeneous sample of community-dwelling adults of the Cam-CAN cohort. We found that sleep quality generally decreases across the lifespan, most strongly for sleep efficiency. However age-related changes in sleep patterns are complex and multifaceted, so we used LCA to identify 'sleep types' associated with specific sleep quality profiles. We found that younger adults are more likely than older adults to display a pattern of sleep problems characterised by poor sleep quality and longer sleep latency, whereas older adults are more likely to display inefficient sleeping, characterised by long periods spent in bed while not asleep. Moreover, the probability of being a 'good' sleeper, unaffected by any adverse sleep symptoms, decreases considerably after age 50 . Notably, closer investigation of the sleep classes reveals 

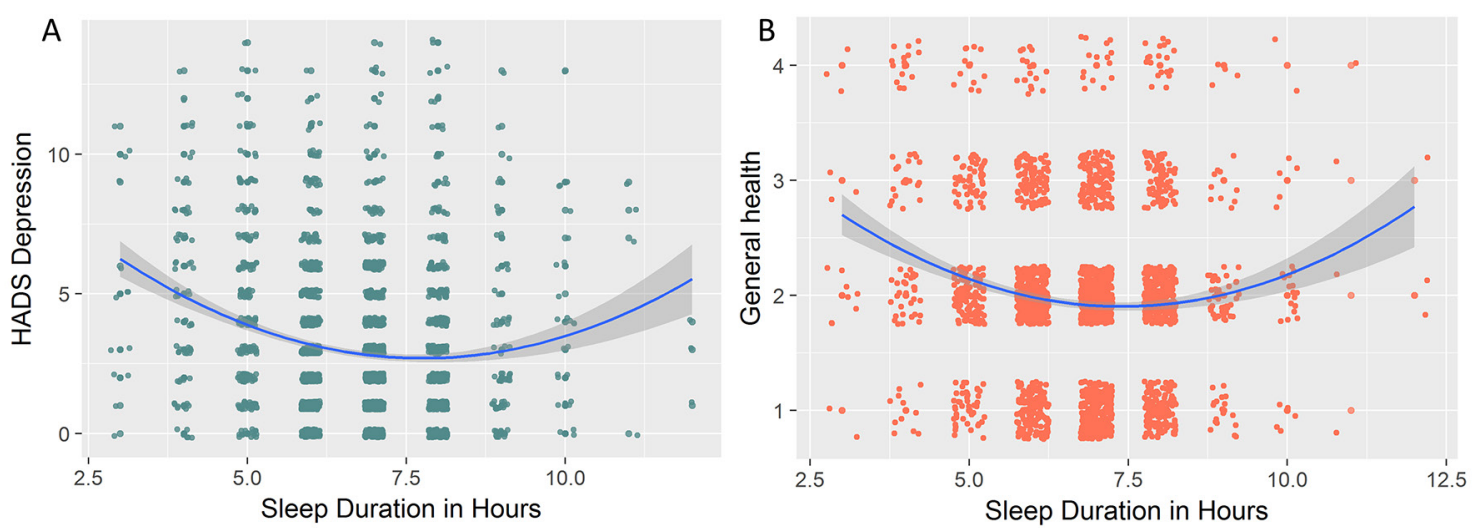

Figure 7 Curvilinear associations between sleep duration in hours and (A) Hospital Anxiety and Depression Scale (HADS) depression and (B) general health (self-reported). For visual clarity, a small amount of random jitter was added to the data points.

likely further complexities of age-related differences. The category 'poor sleepers', most prevalent in older adults, shows high conditional likelihood of 'poor sleep' across all symptoms except 'daytime dysfunction'. One possible explanation is that almost all individuals in this group are beyond retirement age. For this reason, they likely have greater flexibility in tailoring their day-to-day activities to their energy levels (as opposed to individuals working full time) and are therefore less likely to consider themselves 'disrupted' even in the presence of suboptimal sleep. Although more detailed, interview-based investigations would be necessary to examine the precise nature of these findings, it stands to reason that certain symptoms change not just in prevalence but also in meaning across the lifespan.

One key strength of our broad phenotypic assessment is that it allows for direct comparison of the different measures of sleep quality and four key health domains. We found strongest associations between sleep quality and mental health, moderate relations between sleep quality and physical health and cognitive health and sleep, virtually all such that poorer sleep is associated with poorer health outcomes. We did not find evidence for associations between self-reported sleep and neural health. Notably, the relationships we observed are mostly stable across the lifespan, affecting younger and older individuals alike. A notable exception to these effects is the absence of any strong relation (after controlling for age) between sleep quality and neural health as indexed by tract-based average fractional anisotropy. Perhaps surprisingly, given the strong relationships in the same sample between sleep and other outcomes (eg, mental health, online supplementary figure 10), we found that self-reported sleep problems in a non-clinical sample are not associated with fractional anisotropy above and beyond old age. This is despite the fact that previous work within the same cohort observed moderate to strong associations between white matter and various cognitive outcomes. ${ }^{42} 8081$ However, although notable, our finding does not rule out that such associations do exist with other white matter metrics, that they would be observed with objective measures of sleep such as polysomnography, or that the co-occurrence of age-related declines in sleep quality and white matter share an underlying causal association that cannot be teased apart in a cross-sectional sample.

One strength of our study is the assessment of neuroimaging metrics, namely, fractional anisotropy, in a large, community-dwelling healthy population. Fractional anisotropy is often used in studies of ageing (eg, Madden $)^{82}$ is relatively reliable ${ }^{83}$ and is sensitive to clinical anomalies such as WMHs. However, the relationship between FA and white matter health is indirect $\mathrm{t}^{408}$ and drawbacks include its inability to distinguish crossing fibres (eg, Jones et $a t^{40}$ and Wandell ${ }^{84}$ ) and vulnerability to movement and the fact that it likely reflects a combination of underlying physiological properties. Various alternative white matter metrics exist, including summary measures of diffusivity (eg, axial/radial/mean diffusivity), volumetric measures of white matter hyperintensity and various innovative measures currently in development, but assessment of their physiological validity is ongoing. ${ }^{84} 85$

While there are limitations of self-report measures including in older cohorts, ${ }^{19}$ including the fact that they likely reflect different aspects of sleep health than polysomnography (sleep in the lab), our results suggest that there are considerable advantages in using self-reported sleep measures: first, obtaining sleep quality data in a large and broadly phenotyped sample is feasible and second, our results demonstrated clear and consistent associations across multiple domains for both subjective (eg, self-reported health) and objective measures (eg, memory tests, BMI), which both replicate and extend previous lab-based sleep findings. Future work should ideally simultaneously measure polysomnography and self-report in longitudinal, large-scale cohorts to fully capture the range of overlapping and complementary relations between different aspects of sleep quality and health outcomes. ${ }^{19}$ 
For both self-report and objective measures of sleep quality, an open question is that of causality: does poor sleep affect health outcomes, do health problems affect sleep, are they both markers of some third problem or do causal influences go both ways? Most likely, all these patterns occur to varying degrees. Previous studies have shown that sleep quality causally affects health outcomes such as diabetes ${ }^{43}$ and memory consolidation, ${ }^{1}$ while other evidence suggests that depression directly affects sleep quality ${ }^{86} 87$ and that damage to neural structures may affect sleep regulation. ${ }^{88}$ Although our findings are in keeping with previous findings, our cross-sectional sample cannot tease apart the causal direction of the observed associations, so more work remains to be done to disentangle these complex causal pathways.

In our paper, we focus on a healthy, age-heterogeneous community-dwelling sample. This allows us to study the associations between healthy ageing and self-reported sleep quality, but comes with two key limitations of the interpretations of our findings. First and foremost, our findings are cross-sectional, not longitudinal. This means that we can make inferences about age-related differences, but not necessarily age-related changes. ${ }^{89}{ }^{90}$ One reason why cross-sectional and longitudinal estimates may diverge is that older adults can be thought of as cohorts that differ from the younger adults in more ways than age alone. For example, our age range includes individuals born in the twenties and thirties of the 20th century. Compared with someone born in the 21st century, these individuals will likely have experienced various differences during early life development (eg, less broadly accessible education, lower quality of healthcare, poorer nutrition and similar patterns). For some of our measures, these are inherent limitations-truly longitudinal study of neural ageing is inherently impossible as scanner technology has not been around sufficiently long. This means our findings likely reflect a combination of effects attributable to age-related changes as well as baseline differences between subpopulations that may affect both mean differences and developmental trajectories.

Second, our sample reflects an atypical population in the sense that they are willing and able to visit the laboratory on multiple occasions for testing sessions. This subsample is likely a more healthy subset of the full population, which will mean the range of (poor) sleep quality as well as (poorer) health outcomes will likely be less extreme than in the full population. However, this challenge is not specific to our sample. In fact, as the Cam-CAN cohort was developed using stratified sampling based on primary healthcare providers, our sample is likely as population representative as is feasible for a cohort of this magnitude and phenotypic breadth (see Shafto $e t a l^{12}$ for further details). Nonetheless, a healthier subsample may lead to restriction of range,${ }^{91}$ that is, an attenuation of the strength of the associations observed between sleep quality and health outcomes. Practically, this means that our results likely generalise to comparable, healthy community-dwelling adults, but not necessarily to populations that include those affected by either clinical sleep deprivation or other serious health conditions.

\section{CONCLUSIONS}

Taken together, our study allows several conclusions. First, although we replicate the age-related deterioration in some aspects of sleep quality, other aspects remain stable or even improve. Second, we show that the profile of sleep quality changes across the lifespan. This is important methodologically, as it suggests that PSQI sum scores do not capture the full picture, especially in age-heterogeneous samples. Moreover, it is important from a psychological standpoint: we show that 'sleep quality' is a multidimensional construct and should be treated as such if we wish to understand the complex effects and consequences of sleep quality across the lifespan. Third, moderate to strong relations exist between sleep quality and cognitive, physical and mental health, and these relations largely remain stable across the lifespan. In contrast, we show evidence that in non-clinical populations, poorer self-reported sleep is not reliably associated with poorer neural health. Finally, we find that for absolute sleep duration, we replicate previous findings that both longer and shorter than average amounts of sleep are in association with poorer self-reported general health and higher levels of depression and anxiety.

Together with previous experimental and longitudinal evidence, our findings suggest that at least some age-related decreases in health outcomes may be due to poorer sleep quality. We show that self-reported sleep quality can be an important indicator of other aspects of healthy functioning throughout the lifespan, especially for mental and general physical health. Our findings suggest that accurate understanding of sleep quality is essential in understanding and supporting healthy ageing across the lifespan.

Correction notice This article has been corrected since it was published online. Author comments, erroneously inserted in the PDF, have been removed.

Twitter @rogierk

Acknowledgements We would like to thank Richard Morey and Eric-Jan Wagenmakers for valuable suggestions regarding the use of the BayesFactor package. We are grateful to the Cambridge Centre for Ageing and Neuroscience respondents and their primary care teams in Cambridge for their participation in this study. We also thank colleagues at the MRC Cognition and Brain Sciences Unit MEG and MRI facilities for their assistance.

Collaborators The Cam-CAN corporate author consists of the project principal personnel: Lorraine K Tyler, Carol Brayne, Edward T Bullmore, Andrew C Calder, Rhodri Cusack, Tim Dalgleish, John Duncan, Richard N Henson, Fiona E Matthews, William D Marslen-Wilson, James B Rowe; Research Associates: Karen Campbell, Teresa Cheung, Simon Davis, Linda Geerligs, Anna McCarrey, Abdur Mustafa, Darren Price, David Samu, Jason R Taylor, Matthias Treder, Kamen Tsvetanov, Janna van Belle, Nitin Williams; Research Assistants: Lauren Bates, Tina Emery, Sharon ErzinÃßlioglu, Sofia Gerbase, Stanimira Georgieva, Claire Hanley, Beth Parkin, David Troy; Affiliated Personnel: Tibor Auer, Marta Correia, Lu Gao, Emma Green, Rafael Henriques; Research Interviewers: Jodie Allen, Gillian Amery, Liana Amunts, Anne Barcroft, Amanda Castle, Cheryl Dias, Jonathan Dowrick, Melissa Fair, Hayley Fisher, Anna Goulding, Adarsh Grewal, Geoff Hale, Andrew Hilton, Frances Johnson, Patricia Johnston, Thea Kavanagh-Williamson, Magdalena Kwasniewska, Alison McMinn, Kim Norman, Jessica Penrose, Fiona Roby, Diane Rowland, John Sargeant, Maggie Squire, Beth Stevens, Aldabra Stoddart, Cheryl Stone, Tracy Thompson, Ozlem 
Yazlik; administrative staff: Dan Barnes, Marie Dixon, Jaya Hillman, Joanne Mitchell, Laura Villis.

Contributors AG, RK and MS designed the study. AG and RAK performed the analyses. CC organised and conducted the data collection. AG, MS and RAK wrote the manuscript. YL provided considerable expertise on sleep and poor sleep outcomes. All authors approved the final manuscript.

Funding The Cambridge Centre for Ageing and Neuroscience (Cam-CAN) research was supported by the Biotechnology and Biological Sciences Research Council (grant number BB/H008217/1). RAK is supported by the Sir Henry Wellcome Trust (grant number 107392/Z/15/Z) and by the UK Medical Research Council Programme (MC-A060-5PR60).

Competing interests None declared.

Ethics approval Ethical approval for the study was obtained from the Cambridgeshire 2 (now East of England-Cambridge Central) Research Ethics Committee (reference: 10/H0308/50).

Provenance and peer review Not commissioned; externally peer reviewed.

Data sharing statement The raw data and analysis code are available upon signing a data sharing request form (see http://www.mrc-cbu.cam.ac.uk/datasets/ camcan/ for more detail).

Open Access This is an Open Access article distributed in accordance with the terms of the Creative Commons Attribution (CC BY 4.0) license, which permits others to distribute, remix, adapt and build upon this work, for commercial use, provided the original work is properly cited. See: http://creativecommons.org/ licenses/by/4.0/

(C) Article author(s) (or their employer(s) unless otherwise stated in the text of the article) 2017. All rights reserved. No commercial use is permitted unless otherwise expressly granted.

\section{REFERENCES}

1. Stickgold R. Sleep-dependent memory consolidation. Nature 2005;437:1272-8.

2. Inoué S, Honda K, Komoda Y. Sleep as neuronal detoxification and restitution. Behav Brain Res 1995;69(1-2):91-6.

3. Xie L, Kang $H, X u Q$, et al. Sleep drives metabolite clearance from the adult brain. Science 2013;342:373-7.

4. D'Ambrosio C, Redline S. Impact of Sleep and Sleep Disturbances on Obesity and Cancer. Redline S, Berger NA, editors. New York, NY: Springer New York, 2014.

5. Crowley K. Sleep and sleep disorders in older adults. Neuropsychol Rev 2011;21:41-53.

6. Ohayon MM, Carskadon MA, Guilleminault C, et al. Meta-analysis of quantitative sleep parameters from childhood to old age in healthy individuals: developing normative sleep values across the human lifespan. Sleep 2004;27:1255-73.

7. Middelkoop HA, Smilde-van den Doel DA, Neven AK, et al. Subjective sleep characteristics of 1,485 males and females aged 50-93: effects of sex and age, and factors related to self-evaluated quality of sleep. J Gerontol A Biol Sci Med Sci 1996;51:M108-M115.

8. Schmidt C, Peigneux P, Cajochen C. Age-related changes in sleep and circadian rhythms: impact on cognitive performance and underlying neuroanatomical networks. Front Neurol 2012;3.

9. Leng Y, Wainwright NW, Cappuccio FP, et al. Self-reported sleep patterns in a British population cohort. Sleep Med 2014;15:295-302.

10. Stanley N. The physiology of sleep and the impact of ageing. Euro Urol Suppl 2005;3:17-23.

11. Briones B, Adams N, Strauss M, et al. Relationship between sleepiness and general health status. Sleep 1996;19:583-8.

12. Shafto MA, Tyler LK, Dixon M, et al. The Cambridge Centre for Ageing and Neuroscience (Cam-CAN) study protocol: a crosssectional, lifespan, multidisciplinary examination of healthy cognitive ageing. BMC Neurol 2014;14:204.

13. Buysse D, Reynolds C, Monk T, Berman S, Kupfer D. the Pittsburgh Sleep Quality Index: a new instrument for Psychiatric Practise and Research, 1988:193-213. pdf.

14. Carpenter JS, Andrykowski MA. Psychometric evaluation of the Pittsburgh Sleep Quality Index. J Psychosom Res 1998;45:5-13.

15. Kang SH, Yoon IY, Lee SD, et al. The impact of sleep apnoea syndrome on nocturia according to age in men. BJU Int 2012;110:E851-6:E851-E856.

16. Lou P, Qin Y, Zhang P, et al. Association of sleep quality and quality of life in type 2 diabetes mellitus: a cross-sectional study in China. Diabetes Res Clin Pract 2015;107:69-76.
17. Mellor A, Waters F, Olaithe M, et al. Sleep and aging: examining the effect of psychological symptoms and risk of sleep-disordered breathing. Behav Sleep Med 2014;12:222-34.

18. Kushida CA, Littner MR, Morgenthaler T, et al. Practice parameters for the indications for PSG-AASM Practice Parameters for the indications for polysomnography and related procedures: an update for 2005. Sleep 2005;28.

19. Landry GJ, Best JR, Liu-Ambrose T. Measuring sleep quality in older adults: a comparison using subjective and objective methods. front aging neurosci. Frontiers 2015;77.

20. Altena E, Vrenken H, Van Der Werf YD, et al. Reduced orbitofrontal and parietal gray matter in chronic insomnia: a voxel-based morphometric study. Biol Psychiatry 2010;67:182-5.

21. Spiegelhalder K, Regen W, Prem M, et al. Reduced anterior internal capsule white matter integrity in primary insomnia. Hum Brain Mapp 2014;35:3431-8.

22. Taylor JR, Williams N, Cusack R, et al. The Cambridge Centre for Ageing and Neuroscience (Cam-CAN) data repository: structural and functional MRI, MEG, and cognitive data from a cross-sectional adult lifespan sample. Neuroimage 2017;144.

23. Folstein MF, Folstein SE, McHugh PR. "Mini-mental state". A practical method for grading the cognitive state of patients for the clinician. J Psychiatr Res 1975;12:189-98.

24. Mioshi E, Dawson K, Mitchell J, et al. The Addenbrooke's Cognitive Examination Revised (ACE-R): a brief cognitive test battery for dementia screening. Int J Geriatr Psychiatry 2006;21:1078-85.

25. Regestein QR, Friebely J, Shifren JL, et al. Self-reported sleep in postmenopausal women. Menopause 2004;11:198-207.

26. Curcio G, Ferrara M, De Gennaro L, loss S. Sleep loss, learning capacity and academic performance. Sleep Med Rev 2006;10:323-37.

27. Ferracioli-Oda E, Qawasmi A, Bloch MH, et al. Meta-analysis: melatonin for the treatment of primary sleep disorders. PLoS One 2013;8:e63773

28. Jean-Louis G, von Gizycki H, Zizi F. Melatonin effects on sleep, mood, and cognition in elderly with mild cognitive impairment. $J$ Pineal Res 1998;25:177-83.

29. Scullin MK, Bliwise DL, Sleep BDL. Sleep, cognition, and normal aging: integrating a half century of multidisciplinary research. Perspect Psychol Sci 2015;10:97-137.

30. Nebes RD, Buysse DJ, Halligan EM, et al. Self-reported sleep quality predicts poor cognitive performance in healthy older adults. $J$ Gerontol B Psychol Sci Soc Sci 2009;64:180-7.

31. Kamba M, Inoue Y, Higami S, et al. Cerebral metabolic impairment in patients with obstructive sleep apnoea: an independent association of obstructive sleep apnoea with white matter change. J Neurol Neurosurg Psychiatry 2001;71:334-9.

32. Harbison J, Gibson GJ, Birchall D, et al. White matter disease and sleep-disordered breathing after acute stroke. Neurology 2003;61:959-63.

33. Macey PM, Kumar R, Woo MA, et al. Brain structural changes in obstructive sleep apnea. Sleep 2008;31:967-77.

34. Ramos AR, Dong C, Rundek T, et al. Sleep duration is associated with white matter hyperintensity volume in older adults: the Northern Manhattan Study. J Sleep Res 2014;23:524-30.

35. Unger MM, Belke M, Menzler K, et al. Diffusion tensor imaging in idiopathic REM sleep behavior disorder reveals microstructural changes in the brainstem, substantia nigra, olfactory region, and other brain regions. Sleep 2010;33:767-73. /pmc/ articles//?report=abstract.

36. Macey PM, Henderson LA, Macey KE, et al. Brain morphology associated with obstructive sleep apnea. Am J Respir Crit Care Med. American Thoracic Society 2002;166:1382-7.

37. Sexton CE, Storsve AB, Walhovd KB, et al. Poor sleep quality is associated with increased cortical atrophy in community-dwelling adults. Neurology 2014;83:967-73.

38. Debette S, Markus HS. The clinical importance of white matter hyperintensities on brain magnetic resonance imaging: systematic review and meta-analysis. BMJ 2010;341:c3666.

39. Mädler B, Drabycz SA, Kolind SH, et al. Is diffusion anisotropy an accurate monitor of myelination? correlation of multicomponent T2 relaxation and diffusion tensor anisotropy in human brain. Magn Reson Imaging 2008;26:874-88. Sep26(7).

40. Jones DK, Knösche TR, Turner R, et al. Fiber count, and other fallacies: the do's and don'ts of diffusion MRI. Vol. 73. Neurolmage 2013:239-54.

41. Maillard P, Fletcher E, Harvey D, et al. White matter hyperintensity penumbra. Stroke 2011;42:1917-22.

42. Kievit RA, Davis SW, Griffiths J, et al. A watershed model of individual differences in fluid intelligence. Neuropsychologia 2016;91:186-98. 
43. Spiegel K, Tasali E, Leproult R, et al. Effects of poor and short sleep on glucose metabolism and obesity risk. Nat Rev Endocrinol 2009;5:253-61. Nature Publishing Group;May5(5).

44. Grandner MA, Jackson NJ, Izci-Balserak B, et al. Social and behavioral determinants of perceived insufficient sleep. Front Neurol 2015;56:112.

45. Leng Y, Wainwright NW, Cappuccio FP, et al. Daytime napping and the risk of all-cause and cause-specific mortality: a 13-year follow-up of a British population. Am J Epidemiol 2014;179:1115-24.

46. Leng Y, Cappuccio FP, Wainwright NW, et al. Sleep duration and risk of fatal and nonfatal stroke: a prospective study and meta-analysis. Neurology 2015;84:1072-9.

47. Hoevenaar-Blom MP, Spijkerman AM, Kromhout D, et al. Sleep duration and sleep quality in relation to 12-year cardiovascular disease incidence: the MORGEN study. Sleep 2011;34:1487-92. Nov34(11).

48. Strine TW, Chapman DP. Associations of frequent sleep insufficiency with health-related quality of life and health behaviors. Sleep Med 2005;6:23-7.

49. Taheri S, Lin L, Austin D, et al. Short sleep duration is associated with reduced leptin, elevated ghrelin, and increased body mass index. PLoS Med 2004;1:e62.

50. Roberts RE, Shema SJ, Kaplan GA, et al. Sleep complaints and depression in an aging cohort: a prospective perspective. $A m \mathrm{~J}$ Psychiatry 2000;157:81-8.

51. Breslau N, Roth T, Rosenthal L, et al. Sleep disturbance and psychiatric disorders: a longitudinal epidemiological study of young adults. Biol Psychiatry 1996;39:411-8. Mar39(6).

52. Kaneita $\mathrm{Y}$, Ohida $\mathrm{T}$, Uchiyama $\mathrm{M}$, et al. The relationship between depression and sleep disturbances: a japanese nationwide general population survey. J Clin Psychiatry 2006;67:196-203.

53. Fried El, Nesse RM. Depression sum-scores don't add up: why analyzing specific depression symptoms is essential. BMC Med 2015;13:72.

54. Novati A, Hulshof HJ, Koolhaas JM, et al. Chronic sleep restriction causes a decrease in hippocampal volume in adolescent rats, which is not explained by changes in glucocorticoid levels or neurogenesis. Neuroscience 2011;190:145-55.

55. Ramsawh HJ, Stein MB, Belik SL, et al. Relationship of anxiety disorders, sleep quality, and functional impairment in a community sample. J Psychiatr Res 2009;43:926-33. Jul43(10).

56. Zigmond AS, Snaith RP. The hospital anxiety and depression scale. Acta Psychiatr Scand 1983;67:361-70.

57. Liang F, Paulo R, Molina G, et al. Mixtures of $\mathrm{g}$ priors for Bayesian Variable selection. J am stat assoc. Taylor \& Francis 2008;1103:410-23.

58. Rouder JN, Morey RD. Default Bayes factors for model selection in regression. Multivariate Behav Res 2012;47:877-903.

59. Wagenmakers EJ. A practical solution to the pervasive problems of $p$ values. Psychon Bull Rev 2007;14:779-804.

60. Wetzels R, Matzke D, Lee MD, et al. Statistical evidence in experimental psychology: an empirical comparison using $855 \mathrm{t}$ tests. Perspect Psychol Sci 2011;6:291-8.

61. Gelman A, Hill J, Yajima M, et al. Why we usually don't have to worry about multiple comparisons. J Res Educ Eff 2012;5:189-211.

62. Jeffreys H. A theory of probability. Oxford University Press: Oxford, 1961.

63. Morey RD, Rouder JN. BayesFactor CRAN, 2015.

64. Team RD. R: a language and environment for statistical computing. Vienna 2013.

65. Linzer DA, Lewis JB. poLCA : an $R$ package for polytomous variable latent class analysis. J Stat Softw 2011;42:10.

66. Rhemtulla M, Brosseau-Liard PÉ, Savalei V. When can categorical variables be treated as continuous? A comparison of robust continuous and categorical SEM estimation methods under suboptimal conditions. Psychol Methods 2012;17:354-73.

67. Schwarz G. Estimating the dimension of a Model. The Annals of Statistics 1978;6:461-4.

68. Dayton CM, Macready GB. Concomitant-variable latent-class models. J Am Stat Assoc 1988;83:173-8 http://www.tandfonline. com/doi/abs/10.1080/01621459.1988.10478584.
69. Wickham H. ggplot2: elegant graphics for data analysis. Springer Science \& Business Media 2009:221 p https://books.google.com/ books?hl=en\& $\mid r=\& i d=b e s-A A A A Q B A J \& p g i s=1$.

70. Miller $\mathrm{E}$. Verbal fluency as a function of a measure of verbal intelligence and in relation to different types of cerebral pathology. $\mathrm{Br}$ $J$ Clin Psychol 1984;23 (Pt 1:53-7.

71. Biesbroek JM, van Zandvoort MJ, Kappelle LJ, et al. Shared and distinct anatomical correlates of semantic and phonemic fluency revealed by lesion-symptom mapping in patients with ischemic stroke. Brain Struct Funct 2016;221:2123-34.

72. Troyer AK, Moscovitch M, Winocur G. Clustering and switching as two components of verbal fluency: evidence from younger and older healthy adults. Neuropsychology 1997;11:138-46.

73. Hua K, Zhang J, Wakana S, et al. Tract probability maps in stereotaxic spaces: analyses of white matter anatomy and tractspecific quantification. Neuroimage 2008;39:336-47.

74. Briones B, Adams N, Strauss M, et al. Relationship between sleepiness and general health status. Sleep 1996;19:583-8. Sep19(7).

75. Cizza G, Skarulis M, Mignot E. A link between short sleep and obesity: building the evidence for causation. Sleep 2005;28:1217-20.

76. Gangwisch JE, Malaspina D, Boden-Albala B, et al. Inadequate sleep as a risk factor for obesity: analyses of the NHANES I. Sleep 2005;28:1289-96. Oct28(10).

77. Grandner MA, Drummond SP. Who are the long sleepers? Towards an understanding of the mortality relationship. Sleep Med Rev 2007;11:341-60.

78. Kaneita Y, Uchiyama M, Yoshiike N, et al. Associations of usual sleep duration with serum lipid and lipoprotein levels. Sleep 2008;31:645-52.

79. Grandner MA, Hale L, Moore M, et al. Mortality associated with short sleep duration: the evidence, the possible mechanisms, and the future. Sleep Med Rev 2010;14:191-203.

80. Kievit RA, Davis SW, Mitchell DJ, et al. Distinct aspects of frontal lobe structure mediate age-related differences in fluid intelligence and multitasking. Nat Commun 2014;5:5658.

81. Henson RN, Campbell KL, Davis SW, et al. Multiple determinants of lifespan memory differences. Sci Rep 2016;6:32527.

82. Madden DJ, Bennett IJ, Song AW. Cerebral white matter integrity and cognitive aging: contributions from diffusion tensor imaging. Neuropsychol Rev 2009;19:415-35.

83. Fox RJ, Sakaie K, Lee JC, et al. A validation study of multicenter diffusion tensor imaging: reliability of fractional anisotropy and diffusivity values. AJNR Am J Neuroradiol 2012;33:695-700.

84. Wandell BA. Clarifying Human White matter. Annu Rev Neurosci 2016;39:103-28.

85. Tournier JD, Mori S, Leemans A. Diffusion Tensor imaging and beyond. Magn Reson Med 2011;65:1532-56.

86. Lustberg L, Reynolds CF, . Depression and insomnia: questions of cause and effect. Sleep Med Rev 2000;4:253-62.

87. Sbarra DA, Allen JJB. Decomposing depression: on the prospective and reciprocal dynamics of mood and sleep disturbances.

88. Lim AS, Ellison BA, Wang JL, et al. Sleep is related to neuron numbers in the ventrolateral preoptic/intermediate nucleus in older adults with and without Alzheimer's disease. Brain 2014;137:2847-61.

89. Raz N, Lindenberger U. Only time will tell: cross-sectional studies offer no solution to the age-brain-cognition triangle: Comment on Salthouse. 2011.

90. Schaie KW. The course of adult intellectual development. Am Psychol;49:304-13.

91. Wiberg M, Sundstrom A. A comparison of two approaches to correction of restriction of range in correlation analysis Practical Assessment Research and Evaluation 14.2009 http://pareonline.net.

92. Wechsler CJ. Wechsler memory scale. 3rd edn. London: Harcourt Assessment, 1999.

93. Cattell RB. Abilities: their structure, growth, and action Boston Houghton-Mifflin, 1971.

94. McGee DL, Liao Y, Cao G, et al. Self-reported health status and mortality in a multiethnic US cohort. Am J Epidemiol 1999;149:41-6.

95. Deurenberg P, Weststrate JA, Seidell JC. Body mass index as a measure of body fatness: age- and sex-specific prediction formulas. Br J Nutr 1991;65:105. 